\title{
The Laser in Glaucoma and Ocular Hypertension (LiGHT) Trial. A multicentre randomised controlled trial: Baseline patient characteristics.
}

Authors: Evgenia Konstantakopoulou ${ }^{1,2}$, Gus Gazzard ${ }^{1,2}$, Victoria Vickerstaff ${ }^{3,4}$, Yuzhen Jiang ${ }^{1,2}$, Neil Nathwani ${ }^{1}$, Rachael Hunter ${ }^{5}$, Gareth Ambler ${ }^{6}$, Catey Bunce ${ }^{1,7,8}$, the LiGHT Trial Study Group*

Corresponding author: Gus Gazzard, NIHR Biomedical Research Centre at Moorfields Eye Hospital NHS Foundation Trust and UCL Institute of Ophthalmology, 162 City Road, EC1V 2PD, London UK, gusgazzard@gmail.com.

1. NIHR Biomedical Research Centre at Moorfields Eye Hospital NHS Foundation Trust, 162 City Road, EC1V 2PD, London, UK

2. Institute of Ophthalmology, University College London, 11-43 Bath St, London EC1V 9EL, London, UK

3. Marie Curie Palliative Care Research Department, UCL Division of Psychiatry, University College London, 6th Floor, Maple House, 149 Tottenham Court Road, London W1T 7NF, UK

4. The Research Department of Primary Care and Population Health, University College London, Rowland Hill Street, London NW3 2PF, UK

5. Priment Clinical Trials Unit, University College London, Royal Free Medical School, Rowland Hill Street, NW3 2PF, London, UK

6. Department of Statistical Science, University College London, Gower Street, London WC1E 6BT, UK

7. London School of Hygiene \& Tropical Medicine, London, UK

8. Department of Primary Care \& Public Health, Kings College London, London, UK

* The LiGHT Trial Study Group: Gareth Ambler, Keith Barton, Rupert Bourne, David Broadway, Catey Bunce, Marta Buszewicz, Amanda Davis, Anurag Garg, David GarwayHeath, Gus Gazzard, Rachael Hunter, Yuzhen Jiang, Evgenia Konstantakopoulou, Sheng Lim, Joanna Liput, Timothy Manners, Stephen Morris, Neil Nathwani, Gary Rubin, Nicholas Strouthidis, Victoria Vickerstaff, Sarah Wilson, Richard Wormald, Haogang Zhu.

Abbreviations: Laser in Glaucoma and Ocular-Hypertension: LiGHT, Selective laser trabeculoplasty: SLT, Primary Open Angle Glaucoma: POAG, Ocular Hypertension: OHT, Treatment in Pursuit of Control: TPC, Quality of Life: QoL, Central Corneal Thickness: CCT, The National Institute of Care Excellence: NICE, Intra-ocular pressure: IOP, Randomised clinical trials: RCT, Health-related quality of Life: HRQL, Early Manifest Glaucoma Trial: EMGT, United States of America: USA, Advanced Glaucoma Intervention Study: AGIS, Collaborative Initial Glaucoma Treatment Study, Ocular Hypertension Treatment Study: OHTS, United Kingdom Glaucoma Treatment Study: UKGTS, European Glaucoma Prevention Study: EGPS, Effectiveness in Angle-closure Glaucoma of Lens Extraction: EAGLE, Glaucoma Utility Index: GUI, Glaucoma Symptom Scale: GSS, Glaucoma Quality of Life-15: GQL-15, Client Service Receipt Inventory: CSRI, Glaucoma Progression Analysis: GPA, European Glaucoma Society: EGS, Maximum medical therapy: MMT, Humphrey Visual Field: HVF, Glaucoma Progression Analysis: GPA, Heidelberg Retinal Tomograph: HRT, Visual field: VF, Standard operating procedures: SOP, Quality-Adjusted Life Years: QALYs, National Health Service: NHS.

Address for reprints: Gus Gazzard, NIHR Biomedical Research Centre at Moorfields Eye Hospital NHS Foundation Trust, London, UK, 162 City Road, EC1V 2PD, London UK. 
Word count: 2479

\section{Number of Tables: 3}

Funding: The trial was funded by National Institute of Health Research Health Technology Assessment Panel (Project reference number 09/104/40) and was sponsored by Moorfields Eye Hospital NHS Foundation Trust. The sponsor or funding organisation had no role in the design or conduct of this research. This report presents independent research commissioned by the NIHR; the views and opinions expressed by authors in this publication are those of the authors and do not necessarily reflect those of the NHS, the NIHR, MRC, CCF, NETSCC, the HTA programme or the Department of Health.

Conflict of Interest: Gus Gazzard, David Garway-Heath, Keith Barton, Richard Wormald, Stephen Morris, Rachael Hunter, Gary S. Rubin, Marta Buszewicz, Gareth Ambler and Catey Bunce have received a grant from the National Institute for Health Research for the submitted work. David Garway-Heath and Richard Wormald have received financial support through the Biomedical Research Centre at Moorfields Eye Hospital and UCL Institute of Ophthalmology. Gus Gazzard received a research grant from Lumenis prior to the submitted work. Gus Gazzard, David Garway-Heath and Keith Barton have conflicts of interest outside the submitted work. There are no other competing interests.

Contributorship: EK wrote the manuscript together with $G G$ and is involved in the acquisition of the data. YJ contributed to the drafting of the paper. VV wa involved in the analysis of the data. NN contributed to the drafting of the paper and is involved in acquisition of the data. $\mathrm{RH}$ contributed to the design of the outcome measures and the data to be collected and was involved in the drafting of the manuscript. GA contributed in the design of the outcome measures and the data to be collected and was involved in the drafting of the manuscript. CB contributed in the design of the outcome measures and the data to be collected and was involved in the drafting of the manuscript. GG led the initial conception and design of the trial and writing the protocol, acquired funding and ethics approval, is the chief investigator of the trial and was a major contributor in writing the manuscript. All authors read and approved the final manuscript. 


\section{SYNOPSIS}

The Laser in Glaucoma and Ocular Hypertension trial is a multicentre randomised controlled trial comparing the health-related quality of life, clinical and cost-effectiveness of drops vs. selective laser trabeculoplasty as a first line treatment. 


\section{ABSTRACT}

Purpose: The Laser in Glaucoma and Ocular-Hypertension (LiGHT) Trial aims to establish whether initial treatment with selective laser trabeculoplasty $(S L T)$ is superior to initial treatment with topical medication for Primary Open Angle Glaucoma (POAG) or Ocular Hypertension (OHT).

Design: LiGHT is a prospective unmasked, multi-centre, pragmatic, randomised controlled trial $(\mathrm{RCT})$.

Participants: 718 previously untreated patients with POAG or OHT were recruited at 6 UK centres between 2012 and 2014.

Methods: Patients were randomised to initial SLT followed by medical therapy or medical therapy without laser. Participants will be monitored for 3 years, according to routine clinical practice. The primary outcome is EQ-5D-5L. Secondary outcomes are treatment pathway cost and cost-effectiveness, Glaucoma Utility Index, Glaucoma Symptom Scale, Glaucoma Quality of Life, pathway effectiveness, visual function, safety and concordance.

Results: A total of 555 patients had POAG and $163 \mathrm{OHT} ; 518$ patients had both eyes eligible. The mean age for POAG patients was 64 years and for OHT 58 years. $70 \%$ of all participants were white. Median IOP for OHT eyes was $26 \mathrm{mmHg}$ and $23 \mathrm{mmHg}$ for POAG eyes. Median baseline VF MD was $-0.81 \mathrm{~dB}$ for OHT eyes and $-2.82 \mathrm{~dB}$ for POAG eyes. There was no difference between POAG and OHT patients on the EQ-5D-5DL; the difference between OHT and POAG on the GUI was -0.02 and on the GQL 1.23.

Conclusions: The LiGHT Trial is the first RCT to compare the two treatment options in a real-world setting. The baseline characteristics of the LiGHT cohort compare well with other landmark glaucoma studies. 


\section{INTRODUCTION}

Glaucoma is recognised as the leading cause of irreversible blindness worldwide. ${ }^{1}$ Intraocular pressure (IOP) is the only modifiable risk factor; laser and topical medication both effectively reduce IOP. ${ }^{2-4}$ Topical medical treatment is associated with questionable longterm acceptability and impaired Health Related Quality of Life (HRQL) due to considerable cost, side effects and numerous hospital visits. ${ }^{5}$

The Laser in Glaucoma and Ocular Hypertension (LiGHT) Trial compares HRQL in patients who started treatment using topical IOP lowering medication to that in patients who were treated with SLT first. LiGHT also compares the clinical and cost-effectiveness of the two pathways. The design of LiGHT has been described elsewhere. ${ }^{6}$ Briefly, LiGHT is a multicentre, randomised clinical trial, unmasked to treatment allocation. Eligible patients with POAG or OHT were randomised to either SLT or medical therapy (drops) as first line treatment. Patients with one or both eyes eligible were treated identically. All measurements influencing treatment escalation decisions were made by observers masked to treatment status. Target IOP, follow-up intervals and treatment escalation decisions were guided by custom written decision-support software using visual field, IOP and disc imaging information to avoid bias in clinical decision-making. Patients were followed up for 3 years.

\section{METHODS}

Eligible patients were identified at the National Health Service (NHS) clinics of six participating centres in the UK from October 2012 through to October 2014 (Appendix 1). Patients had newly diagnosed, untreated POAG in one or both eyes (including normal tension glaucoma and pseudoexfoliative glaucoma) or OHT qualifying for treatment according to NICE guidelines, ${ }^{7}$ open angles, and for POAG visual field loss with mean deviation (VF MD) not worse than $-12 \mathrm{~dB}$ in the better eye or $-15 \mathrm{~dB}$ in the worse eye and corresponding damage to the optic nerve head. Patients were 18 years or older and able to understand English, had a visual acuity of 6/36 or better in the treated eye(s), no history of treatment for POAG or OHT and no previous intra-ocular surgery, except uncomplicated phaco-emulsification at least one year before entering the trial. Patients were excluded if there were contra-indications to SLT, they were unable to use topical medical therapy, they had visually significant cataract, or were having treatment for another ophthalmic condition. A glaucoma sub-specialist fellowship-trained consultant ophthalmologist's decision to initiate treatment was required for inclusion in the Trial.

The IOP was measured with Goldman applanation tonometry, by experienced clinicians masked to treatment allocation. The VF tests were performed with the Humphrey Field Analyser Mark II (SITA standard 24-2) (Carl Zeiss Meditec, Dublin, CA). Optic nerve head scans were obtained with the Heidelberg retina tomograph (HRT) (Heidelberg Engineering, Heidelberg, Germany). The refractive error was calculated as the spherical diopters measured with an autorefractor. General health information and medication was recorded based on the patients' reporting.

The study adheres to the tenets of the Declaration of Helsinki and had been granted ethical approval.

\section{Disease classification}

The NICE recommended thresholds were used for disease classification and for initiating treatment. ${ }^{7}$ For the purposes of disease classification 'per patient', participants were classified as having POAG in at least one eye, as this represents the pragmatic treatment in a clinical setting. Patients were classified as having OHT if at least one eye had OHT (but no POAG). 


\section{Outcome Measures}

The primary outcome measure is HRQL measured using EQ-5D-5 $\mathrm{L}^{8}$ with utility scores calculated using the English Time Trade Off (TTO) value set. ${ }^{9}$ Secondary outcome measures are treatment pathway health care resource use, cost and cost-effectiveness [Client Service Receipt Inventory' (CSRI)] questionnaire, ${ }^{10}$ glaucoma specific treatmentrelated quality of life $\left[(\text { Glaucoma Utility Index (GUI) }]^{11}\right.$ patient reported disease and treatment related symptoms [Glaucoma Symptom Scale (GSS)], ${ }^{12}$ and visual function [Glaucoma Quality of Life - 15 (GQL-15) $]^{13}$, objective measurements of pathway effectiveness for IOP lowering and visual function preservation, objective safety measures for each treatment pathway and concordance. Questionnaires are sent to patients at six-monthly intervals.

\section{Statistical analysis}

Summary measures for the baseline characteristics of the participating patients and eyes are presented as mean and standard deviation for continuous variables with a symmetric distribution, medians and inter-quartile ranges for continuous, skewed variables and frequencies and percentages for categorical variables. Means and standard deviations are also reported in some cases and for EQ-5D-5L and GUI to allow for comparisons with values reported in other studies. The difference in means and corresponding $95 \%$ confidence intervals between OHT and POAG in EQ-5D-5L utility scores, GUI, GSS and GQL are generated using linear regression and bootstrapping with 1,000 replications. These summaries are based on observed data only and the number of missing observations will be reported. Stata 13.0 (StataCorp LP, Texas, USA) was used for the data analysis.

\section{RESULTS}

\section{Baseline Patient Characteristics}

718 patients were recruited: 301 patients (41.9\%) had bilateral POAG; 161 (22.4\%) had unilateral POAG (fellow eye healthy); 93 patients (13.0\%) had POAG in one eye and OHT in the other eye; 124 patients (17.3\%) had bilateral OHT and 39 patients $(5.4 \%)$ had unilateral OHT. A total of 555 patients $(77.2 \%)$ were classified as POAG and 163 patients $(22.7 \%)$ were classified as OHT: 518 patients $(72.1 \%)$ had both eyes eligible for the trial; 96 patients $(13.4 \%)$ had only the right eye eligible and 104 patients (14.5\%) had only the left eye eligible; $55 \%$ of the better eyes were right eyes. Table 1 summarises the baseline patient characteristics.

\begin{tabular}{lllll}
\hline & & \multicolumn{1}{c}{ All } & Diagnosis \\
\hline & & $\mathrm{N}$ & $\mathrm{OHT}$ & $\mathrm{POAG}$ \\
& & $(\%) \mathrm{n}=718$ & $\mathrm{n}=163$ & $\mathrm{n}=555$ \\
\hline Centres & Queen's University Belfast & $30(4)$ & $5(3)$ & $25(5)$ \\
& Guy's and St Thomas' Hospital & $106(15)$ & $55(34)$ & $51(9)$ \\
& Huntingdon Hospital & $82(11)$ & $14(9)$ & $68(12)$ \\
& Moorfields Eye Hospital & $374(52)$ & $73(45)$ & $301(54)$ \\
& Norfolk and Norwich University & $89(12)$ & $13(8)$ & $76(14)$ \\
& Hospital & & & $34(6)$ \\
\hline Gender & York Hospital & $37(5)$ & $3(2)$ & $252(45)$ \\
& Memale & $321(45)$ & $69(42)$ & $303(55)$ \\
\hline Age & Meale & $397(55)$ & $94(58)$ & $64(12)$ \\
\hline Ethnicity ${ }^{2}$ & Asian & $63(12)$ & $58(11)$ & $43(8)$ \\
& Black & $51(7)$ & $8(5)$ & $101(18)$ \\
& Whites & $146(20)$ & $45(28)$ & $397(71)$ \\
& Other & $501(70)$ & $104(64)$ & $14(3)$ \\
\hline
\end{tabular}




\begin{tabular}{|c|c|c|c|c|}
\hline \multirow[t]{10}{*}{ General health } & Asthma & $93(13)$ & $17(10)$ & $76(14)$ \\
\hline & Hypertension & $251(35)$ & $60(37)$ & $191(34)$ \\
\hline & Diabetes & $82(11)$ & $24(15)$ & $58(10)$ \\
\hline & Angina & $21(3)$ & $4(2)$ & $17(3)$ \\
\hline & Cardiac Arrhythmia & $37(5)$ & $4(2)$ & $33(6)$ \\
\hline & Ischaemic Heart Disease & $20(3)$ & $3(2)$ & $17(3)$ \\
\hline & CVA/Stroke & $14(2)$ & $2(1)$ & $12(2)$ \\
\hline & Migraines & $94(13)$ & $16(10)$ & $78(14)$ \\
\hline & Peripheral vasospastic symptoms & $63(9)$ & $7(4)$ & $56(10)$ \\
\hline & Blood Loss/Transfusion & $76(11)$ & $14(9)$ & $62(11)$ \\
\hline \multicolumn{2}{|c|}{$\begin{array}{l}\text { Family Ocular History of Glaucoma }{ }^{\mathrm{b}} \\
\text { (1st degree relative) }\end{array}$} & $214(30)$ & $46(28)$ & $168(30)$ \\
\hline
\end{tabular}

In both diagnostic groups, more male than female patients were recruited (POAG 55\% males; OHT 58\% males). The mean age for POAG patients was 64 years ( $\mathrm{SD}=12$ years) (median (IQR) = 65 (56 to 73) and for OHT 58 years (SD=11 years) (median (IQR) = 57 (50 to 65$)$. A total of $70 \%$ of all participants were white; black was the second largest ethnic group (20\%). $30 \%$ of POAG patients reported a family history of glaucoma, compared to $28 \%$ of OHT patients. Systemic hypertension was noted in 34\% of the POAG patients and $37 \%$ of the OHT patients. Use of systemic antihypertensive medication was noted in $35 \%$ of all patients, $27 \%$ were on statins and $11 \%$ were smokers.

\section{Baseline Characteristics for Eligible Eyes}

A total of 854 eyes $(69 \%)$ were classified as POAG and 380 eyes $(31 \%)$ were classified as $\mathrm{OHT}$. The median baseline VF MD was $-0.81 \mathrm{~dB}$ for OHT eyes (mean (SD) $=-1.25(2.05) \mathrm{dB}$ ) and $-2.82 \mathrm{~dB}$ for POAG eyes (mean $(\mathrm{SD})=3.81$ (3.68) $\mathrm{dB}$ ). The median IOP for OHT eyes was $26 \mathrm{mmHg}$ (mean (SD) $=26.7(3.5) \mathrm{mmHg}$ ) and $23 \mathrm{mmHg}$ for POAG eyes (mean (SD) = $23.5(5.4) \mathrm{mmHg}$ ). A total of $24 \%$ of the POAG eyes had a baseline IOP of $19 \mathrm{mmHg}$ or below. Median neuro-retinal rim area was $1.25 \mathrm{~mm}^{2}$ for OHT eyes and $1.04 \mathrm{~mm}^{2}$ for POAG eyes. Median CCT for OHT eyes was $557 \mu \mathrm{m}$ (mean CCT (SD) $=557(39) \mu \mathrm{m}$ ) and for POAG eyes $549 \mu \mathrm{m}$ (mean CCT (SD) $=549$ (36) $\mu \mathrm{m}$ ). Glaucomatous eyes were more myopic and had worse VA than OHT eyes (Table 2).

\begin{tabular}{|c|c|c|c|}
\hline & All eyes $(N=1234)$ & OHT (n= 380) & POAG $(n=854)$ \\
\hline & \multicolumn{3}{|c|}{ Median (IQR) } \\
\hline $\begin{array}{l}\text { Refractive Error }{ }^{a} \\
\text { (Spherical D) }\end{array}$ & $0.25(-1.25$ to 1.5$)$ & $0.44(-0.75$ to 1.5$)$ & $0.25(-1.5$ to 1.5$)$ \\
\hline $\begin{array}{l}\text { Visual acuity } \\
\text { VF MD (dB) } \\
\text { VF PSD (dB) } \\
\text { HRT Rim Area } \\
\text { IOP } \\
\text { CCT }(\mu \mathrm{m})^{\text {b }} \\
\text { PXF, } n(\%)\end{array}$ & $\begin{array}{l}0.04(-0.04 \text { to } 0.14) \\
-2.15(-4.46 \text { to }-0.57) \\
2.42(1.71 \text { to } 4.73) \\
1.13(0.91 \text { to } 1.37) \\
24(21 \text { to } 28) \\
551 \text { (527 to } 576) \\
17 \text { (1) }\end{array}$ & $\begin{array}{l}0.02(-0.08 \text { to } 0.12) \\
-0.81(-2.4 \text { to } 0.28) \\
1.74(1.465 \text { to } 2.295) \\
1.25(1.09 \text { to } 1.53) \\
26(24 \text { to } 29) \\
557(531 \text { to } 580) \\
9(2)\end{array}$ & $\begin{array}{l}0.06(-0.02 \text { to } 0.16) \\
-2.82(-5.53 \text { to }-1.16) \\
3.08(1.99 \text { to } 6.21) \\
1.04(0.83 \text { to } 1.29) \\
23(20 \text { to } 27) \\
549(524 \text { to } 573) \\
8(1)\end{array}$ \\
\hline $\begin{array}{l}\text { Phacoemulsification in } \\
\text { the past, } \mathrm{n}(\%)\end{array}$ & $72(6)$ & $7(2)$ & $65(8)$ \\
\hline
\end{tabular}

Table 21234 eyes of 718 patients were treated in the study. a: data available for 713 patients; b: data available for 715 patients. VF MD- Visual field Mean Deviation; VF PSD- visual field pattern standard 
deviation; HRT- Heidelberg Retina Tomograph; IOP- Intraocular pressure; CCT- Central corneal thickness; PXF- Pseudoexfoliation; IQR- Inter-quartile range.

\section{Baseline Data of Questionnaire Survey}

POAG patients scored a median of 0.94 on the EQ-5D-5DL (mean (SD) $=0.92(0.13)$ ) and 0.90 on GUI (mean (SD) $=0.89(0.12)$ ). On GSS POAG patients scored a median of 85 $($ mean $(\mathrm{SD})=82.4(16.7)$ ) and on GQL-15 17 (mean $(\mathrm{SD})=19.1(6.3))$. OHT patients scored a median of 0.94 on the EQ-5D-5DL (mean (SD) $=0.91(0.14)$ ) and 0.93 on GUI (mean (SD) $=0.91(0.1)$ ). On GSS OHT patients scored a median of 85 (mean (SD) $=82.3(17.6)$ ) and on GQL-15 16 (mean (SD) = 17.9 (5.4)) (Table 3). There was no difference between POAG and OHT patients on the EQ-5D-5L (difference $0.00,95 \%$ confidence interval $(\mathrm{Cl})-0.02$ to 0.03 ) and on the GSS (difference $0.08,95 \% \mathrm{Cl}-2.91$ to 3.07); the difference between OHT and POAG on the GUI was $-0.02(95 \% \mathrm{Cl}-0.04$ to 0.001$)$ and on the GQL difference 1.23 $(95 \% \mathrm{Cl} 0.15$ to 2.31$))$.

\begin{tabular}{|c|c|c|}
\hline & $\begin{array}{l}\text { OHT } \\
(n=163)\end{array}$ & $\begin{array}{l}\text { POAG } \\
(n=554)\end{array}$ \\
\hline & \multicolumn{2}{|c|}{ Median (IQR) } \\
\hline EQ-5D-5L Index & $0.94(0.89,1.00)$ & $0.94(0.88,1.00)$ \\
\hline Glaucoma Utility Index a & $0.93(0.87,1.00)$ & $0.90(0.81,1.00)$ \\
\hline Glaucoma Symptom Scale ${ }^{b}$ & $85(75,95)$ & $85(73,95)$ \\
\hline Symptom & $83(71,100)$ & $83(71,96)$ \\
\hline Function & $88(75,100)$ & $88(75,100)$ \\
\hline Glaucoma Quality of Life-15 ${ }^{\mathrm{C}}$ & $16(15,19)$ & $17(15,21)$ \\
\hline Central & $2(2,3)$ & $2(2,3)$ \\
\hline Peripheral & $7(7,8)$ & $7(7,9)$ \\
\hline Dark & $6(6,8)$ & $7(6,9)$ \\
\hline Outdoor & $1(1,1)$ & $1(1,1)$ \\
\hline
\end{tabular}

Table 3: Baseline values for EQ5D-5L, Glaucoma Utility Index, Glaucoma Utility Scale, Glaucoma Quality of Life-15; a: $n=716, b: n=710, c: n=712$.

\section{DISCUSSION}

The LiGHT Trial is a multi-centre RCT, comparing HRQL, clinical- and cost-effectiveness and safety of SLT versus topical IOP lowering medication in treatment-naïve patients with newly diagnosed POAG or OHT. LiGHT has an eye specific Target IOP and follows routine clinical practice, permitting any medication (apart from pilocarpine) and any treatment escalations, providing a realistic analysis of glaucoma and OHT management.

The higher black population in LiGHT (20\%) compared to the United Kingdom Glaucoma Treatment Study (UKGTS) $(5.2 \%)^{14}$ is attributable to the high proportion recruited in ethnically diverse London (66.8\%), similar to studies conducted in the USA, such as the Advanced Glaucoma Intervention Study (AGIS), ${ }^{15}$ Collaborative Glaucoma Initial Glaucoma Treatment Study (CIGTS) ${ }^{16}$ and Ocular Hypertension Treatment Study (OHTS), ${ }^{17}$ with higher proportions of black participants $(56.2 \%, 38.1 \%, 25 \%$, respectively).

A total of $42 \%$ of the patients had bilateral POAG, a proportion lower than UKGTS $(51.8 \%)$, but higher than the Early Manifest Glaucoma Trial (EMGT) (24\% overall). ${ }^{18}$

\section{Clinical characteristics}


LiGHT POAG patients were slightly younger than the UKGTS cohort (mean 64.1 and 66 years, respectively). The EMGT recruited older newly-diagnosed patients (median 65 years for LiGHT POAG and 68 years for EMGT), whereas CIGTS recruited younger patients (median 56 and 61 years for black and white patients, respectively). Age differences might reflect the differing proportion of black patients, in whom POAG is seen at a younger age. There were more male patients in the LiGHT POAG cohort (55\%), as in the UKGTS $(52.9 \%)$ and CIGTS (55\%).

The LiGHT POAG cohort had overall higher IOPs than UKGTS (mean IOP 23.5 vs $19.5 \mathrm{mmHg}$, respectively) and EMGT (median LiGHT $23 \mathrm{mmHg}$ vs EMGT 20.5mm Hg); CIGTS reported distinctly higher IOPs (mean $27.6 \mathrm{mmHg}$ medicine group, $27.4 \mathrm{mmHg}$ surgery group). Nearly a quarter of POAG LiGHT eyes had a baseline IOP $\leq 19 \mathrm{mmHg}$, fewer than in the EMGT $(56.1 \%)$ and UKGTS (43\%). Differences in mean presenting IOP between studies may reflect differences between referral patterns, with a more frequent rate of normal tension glaucoma (NTG) diagnosis in some areas.

LiGHT POAG patients appeared to have early stage disease similar to UKGTS (median VF MD -2.8dB for LiGHT, UKGTS -2.9dB), and less severe than EMGT (median VF MD -4.7dB) and CIGTS $(-5.5 \mathrm{~dB})$, possibly reflecting different referral thresholds or access to screening. Similarly to UKGTS and CIGTS, 30\% of LiGHT POAG patients reported having a family history of POAG.

Systemic hypertension was noted in 34\% of the LiGHT POAG patients, similar to EMGT $(38 \%)$ and CIGTS (37\% overall), but lower than UKGTS (57.8\%) and AGIS (overall $46.78 \%)$. Diabetes was recorded for $11 \%$ of the LiGHT patients, similar to the UKGTS (10.5\%), but different to studies conducted in other countries (AGIS 20.3\%, EMGT 4\%). A total of $3 \%$ of LiGHT POAG patients reported ischemic heart disease (UKGTS 5.4\%, EMGT 6\%). Almost a quarter of the POAG patients reported symptoms suggestive of vasospastic conditions, (LiGHT POAG 10\%, EMGT 9\%) or migraines (LiGHT POAG 14\%, EMGT 10\%), compared to almost half of the UKGTS patients (overall 47.4\%).

The LiGHT OHT cohort was on average slightly older than the OHTS cohort (mean LiGHT 57.7, OHTS 55.4 years), but similar to the European Glaucoma Prevention Study (EGPS) cohort. ${ }^{19}$ The LiGHT OHT cohort also had more males $(58 \%)$ than females, unlike OHTS and EGPS.

OHT eyes of the LiGHT cohort had a mean IOP of $26.7 \mathrm{mmHg}$, higher than that reported by OHTS $(24.9 \mathrm{mmHg})$ and EGPS $(23.6 \mathrm{mmHg})$. LiGHT OHT eyes had worse VF MD (mean $1.25 \mathrm{~dB})$ than the OHTS cohort $(0.24 \mathrm{~dB})$ and the EGPS cohort $(0.18 \mathrm{~dB})$. CCT in the LiGHT OHT cohort was lower than the OHTS cohort (mean 557 vs $573 \mu \mathrm{m}$, respectively). These differences could arise from differences between NICE treatment thresholds and OHTS entry criteria.

Of the LiGHT OHT patients $28 \%$ reported a family history of glaucoma, in contrast to $44 \%$ of the OHTS cohort. The prevalence of systemic hypertension and diabetes in the LiGHT OHT cohort were similar to OHTS.

The mean IOP was lower in POAG eyes compared to OHT eyes $(23.5$ vs $26.7 \mathrm{mmHg}$, respectively). OHT eyes had overall thicker corneas compared to POAG eyes (557 vs $549 \mu \mathrm{m}$, respectively). More POAG patients reported symptoms suggestive of vasospastic conditions compared to OHT patients.

\section{HRQL}

The EQ-5D-5L was developed to overcome some of the ceiling effects commonly seen in the EQ-5D-3L, having more discriminatory power and less of a ceiling effect. ${ }^{20}$ This is the 
only study, after EAGLE, to report the results of the EQ-5D-5L in a UK population with OHT or POAG.

The mean EQ-5D-5L score of 0.92 for the LiGHT POAG cohort is higher than the UK average EQ-5D-3L score of 0.799 to $0.779 .{ }^{21}$ LiGHT patients diagnosed with POAG had lower scores than newly diagnosed EAGLE patients, despite the previous exposure of the latter cohort to IOP lowering treatment. ${ }^{22}$ The utility scores of the LiGHT POAG cohort are higher than other cohorts of POAG patients, although these are for older patients with more advanced disease or under various treatment modalities. ${ }^{23}$

Mean GUI scores of the POAG LiGHT cohort (0.89) were not substantially different from the EAGLE study (lens extraction 0.897, peripheral iridotomy 0.921 ).$^{22}$ LiGHT POAG patients scored higher in the GSS than patients in other studies. ${ }^{12}$ LiGHT POAG patients showed better QoL compared to other POAG cohorts as measured with the GQL-15 and scored similarly to patients with normal VF. ${ }^{24}$

HRQL has not been studied extensively in OHT patients. In OHTS, the recruited patients showed better HRQL at baseline than age- and gender-matched population based norms, which was attributed to the cohort's high educational and/or socioeconomic status. The LiGHT OHT cohort shows higher utility scores, measured with the EQ-5D-5DL and GQL-15, when compared to a different cohort of untreated $\mathrm{OHT} .{ }^{25}$

There was no difference between POAG and OHT patients on EQ-5D-5L and the GSS, but GUI, a preference based tool to measure disutility directly related to glaucoma used by the EAGLE trial, and GQL were both better at discriminating between OHT and POAG, even at an early disease stage, suggesting greater sensitivity in this setting.

\section{Acknowledgements}

We are grateful to Emily Dowse, Karine Girard-Claudon, Seetal Savania-Dholakia, Gurveen Panesar, Ayse Barnes, Dominic Carrington, Emerson Tingco, Charles Amoa, Kanom Bibi.

\section{Competing interests}

Gus Gazzard, David Garway-Heath, Keith Barton, Richard Wormald, Stephen Morris, Rachael Hunter, Gary S. Rubin, Marta Buszewicz, Gareth Ambler and Catey Bunce have received a grant from the National Institute for Health Research for the submitted work. David Garway-Heath and Richard Wormald have received financial support through the Biomedical Research Centre at Moorfields Eye Hospital and UCL Institute of Ophthalmology. Gus Gazzard received a research grant from Lumenis prior to the submitted work. Gus Gazzard, David Garway-Heath and Keith Barton have conflicts of interest outside the submitted work. There are no other competing interests.

\section{Funding}

The trial was funded by National Institute of Health Research Health Technology Assessment Panel (Project reference number 09/104/40) and was sponsored by Moorfields Eye Hospital NHS Foundation Trust. The sponsor or funding organisation had no role in the design or conduct of this research. This report presents independent research commissioned by the NIHR; the views and opinions expressed by authors in this publication are those of the authors and do not necessarily reflect those of the NHS, the NIHR, MRC, CCF, NETSCC, the HTA programme or the Department of Health. 
The full protocol can be accessed at:

https://njl-admin.nihr.ac.uk/document/download/2010005.

Further information is available at:

https://www.journalslibrary.nihr.ac.uk/programmes/hta/0910440/\#/ 


\section{Reference}

1. Bunce $C$, Xing W, Wormald R. Causes of blind and partial sight certifications in England and Wales: April 2007-March 2008. Eye (Lond). 2010;24:1692-9.

2. The Advanced Glaucoma Intervention Study (AGIS): 7. The relationship between control of intraocular pressure and visual field deterioration. The AGIS Investigators. Am J Ophthalmol. 2000;130:429-40.

3. Leske MC, Heijl A, Hussein M, et al. Factors for glaucoma progression and the effect of treatment: the early manifest glaucoma trial. Archives of ophthalmology. 2003;121:48-56.

4. Kass MA, Heuer DK, Higginbotham EJ, et al. The Ocular Hypertension Treatment Study: a randomized trial determines that topical ocular hypotensive medication delays or prevents the onset of primary open-angle glaucoma. Arch Ophthalmol. 2002;120:701-13; discussion 829-30.

5. Friedman DS, Okeke CO, Jampel HD, et al. Risk factors for poor adherence to eyedrops in electronically monitored patients with glaucoma. Ophthalmology. 2009;116:1097-105.

6. Gazzard G, Konstantakopoulou E, Garway-Heath D, et al. The Laser in Glaucoma and Ocular Hypertension Trial. A multicentre randomised controlled trial: Design and methodology. Manuscript submitted for publication. 2017.

7. National Institute for Health and Clinical Excellence. NICE: Guidance on Glaucoma: Diagnosis and management of chronic open angle glaucoma and ocular hypertension: $\mathrm{DoH}$; 2010 [Available from: www.nice.org.uk/CG85fullguideline.

8. Herdman M, Gudex C, Lloyd A, et al. Development and preliminary testing of the new five-level version of EQ-5D (EQ-5D-5L). Qual Life Res. 2011;20:1727-36.

9. Devlin N, Shah K, Feng Y, et al. Valuing Health-Related Quality of Life: An EQ-5D-5L Value Set for England. Office of Health Economics. 2016.

10. Patel A, Rendu A, Moran P, et al. A comparison of two methods of collecting economic data in primary care. Fam Pract. 2005;22:323-7.

11. Burr JM, Kilonzo M, Vale L, et al. Developing a preference-based Glaucoma Utility Index using a discrete choice experiment. Optom Vis Sci. 2007;84:797-808.

12. Lee BL, Gutierrez P, Gordon M, et al. The Glaucoma Symptom Scale. A brief index of glaucoma-specific symptoms. Arch Ophthalmol. 1998;116:861-6.

13. Nelson P, Aspinall P, Papasouliotis $O$, et al. Quality of life in glaucoma and its relationship with visual function. J Glaucoma. 2003;12:139-50.

14. Lascaratos G, Garway-Heath DF, Burton R, et al. The United Kingdom Glaucoma Treatment Study: a multicenter, randomized, double-masked, placebo-controlled trial: baseline characteristics. Ophthalmology. 2013;120:2540-5.

15. The Advanced Glaucoma Intervention Study (AGIS): 3. Baseline characteristics of black and white patients. Ophthalmology. 1998;105:1137-45. 
16. Musch DC, Lichter PR, Guire KE, et al. The Collaborative Initial Glaucoma Treatment Study: study design, methods, and baseline characteristics of enrolled patients. Ophthalmology. 1999;106:653-62.

17. Gordon MO, Kass MA. The Ocular Hypertension Treatment Study: design and baseline description of the participants. Arch Ophthalmol. 1999;117:573-83.

18. Leske MC, Heijl A, Hyman L, et al. Early Manifest Glaucoma Trial: design and baseline data. Ophthalmology. 1999;106:2144-53.

19. Miglior S, Zeyen T, Pfeiffer N, et al. The European glaucoma prevention study design and baseline description of the participants. Ophthalmology. 2002;109:1612-21.

20. Janssen MF, Pickard AS, Golicki D, et al. Measurement properties of the EQ-5D-5L compared to the EQ-5D-3L across eight patient groups: a multi-country study. Qual Life Res. 2013;22:1717-27.

21. Szende A, Janssen B, Cabases J, editors. Self-Reported Population Health: An International Perspective based on EQ-5D. London: Springer Open; 2014.

22. Azuara-Blanco A, Burr J, Ramsay C, et al. Effectiveness of early lens extraction for the treatment of primary angle-closure glaucoma (EAGLE): a randomised controlled trial. Lancet. 2016;388:1389-97.

23. Aspinall PA, Johnson ZK, Azuara-Blanco A, et al. Evaluation of quality of life and priorities of patients with glaucoma. Invest Ophthalmol Vis Sci. 2008;49:1907-15.

24. Goldberg I, Clement $\mathrm{Cl}$, Chiang $\mathrm{TH}$, et al. Assessing quality of life in patients with glaucoma using the Glaucoma Quality of Life-15 (GQL-15) questionnaire. J Glaucoma. 2009;18:6-12.

25. van Gestel A, Webers CA, Beckers $\mathrm{HJ}$, et al. The relationship between visual field loss in glaucoma and health-related quality-of-life. Eye (Lond). 2010;24:1759-69. 